\title{
Quick Dr: A Smart Mobile Application for Health Care
}

\author{
Bhumica M J, Inchara S, M R Spoorthi Raj, Neha M R, P Raviraj* \\ Department of CSE, GSSSIETW, Mysuru, Karnataka, India
}

DOI: https://doi.org/10.21467/proceedings.1.6

* Corresponding author email: raviraj@gsss.edu.in

\begin{abstract}
The main aim of the project is to develop a mobile application using Android Studio. This application exhibits sufficient information about the medical attention requested by the user. The application collects details about the ailment which needs to be attended to, and accordingly suggests the procedure to be followed to avail better treatment. The merit of this application is that it collects the current location of the user and displays the suggestions within the specific area of the user. The user can also give a location of his choice and look for the available services in that area. This project results in creating a user-friendly mobile application, where-in the users can browse through lists of hospitals and doctors, schedule appointments, request ambulance service and much more.

Index Terms- Android-Studio, Firebase Database;
\end{abstract}

\section{INTRODUCTION}

Nowadays, health condition of people is degrading at a very high rate. Ever changing life style, bad food habits and increased stress levels tend to affect public health. This has resulted in people becoming more health conscious. In requirement of medical attention people use many applications that provide the services. We are designing an application using Android platform to cater the medical needs of the user with greater benefits. This application fetches the current location of the user and provides information about the services required by them which are nearest to their location. The services include Doctors, Hospitals, Pharmacy, Pathology, Ambulance and Blood bank.

\section{LITERATURE SURVEY}

Many applications related to health care have been developed which are available online.

Few examples include:

$>$ Mfine - Consult top doctors online[1]

$>$ DocsApp - Consult Doctor Online 24*7 on chat/call[2]

$>$ Practo - Doctors, Order Medicines, Consult Online[3]

$>1 \mathrm{mg}-$ Medicines, Health Tests, Doctor Consultation [4]

$>$ Lybrate - Consult a Doctor[5]

$>$ Zoylo - Doctors, Diagnostics, Order Medicines[6]

(C) 2018 Copyright held by the author(s). Published by AIJR Publisher in Proceedings of the $3^{\text {rd }}$ National Conference on Image Processing, Computing, Communication, Networking and Data Analytics (NCICCNDA 2018), April 28, 2018.

This is an open access article under Creative Commons Attribution-NonCommercial 4.0 International (CC BY-NC 4.0) license, which permits any non-commercial use, distribution, adaptation, and reproduction in any medium, as long as the original work is properly cited. ISBN: 978-81-936820-0-5 
- mfine: mfine app is a digital health platform that aggregates the best hospitals and their doctors from your city and nearby area to provide on-demand healthcare services. mfine will be launching following new healthcare services on the application.

- Practo: Practo makes finding great doctors and booking appointments with them, super easy. By entering the symptoms and instantly see a list of verified doctors with detailed information including their medical qualifications, experiences, fee etc.

- Lybrate: Medical platform connecting over top Doctors and pathology labs. User can ask a free question about any health or fitness query and get multiple helpful answers from the best doctors within a few minutes.

- Zoylo: Zoylo is online healthcare app that connects users to a plethora of healthcare services like online doctor appointment, diagnostic booking, order medicines, and more in the most convenient and hassle-free way.

\section{PROPOSED METHOD}

This is an android based application, so we are using Android-Studio to design the application and firebase for storing of data to the server.

\section{Android-Studio:}

Android-Studio is official IDE of Android. It is built purposefully for Android to accelerate the user development and help the user or anyone to build the highest-quality applications for Android device. It consists of many tools which is offers custom-tailored for the Android application developers. Those are editing, debugging, testing and profiling tools. [7]

\section{Firebase:}

The best feature of the firebase is real time database is a cloud-hosted database. Data is stored as JSON and it can be synchronized in a real time to every connected client. Users can build cross-platforms applications with iOS, Android, and JavaScript SDKs, all of the users can share one single real time database instance and automatically receive updates and can fetch the newest data. [8]

We are proposing a smart Mobile application called "QUICK Dr" by using those two tools as mentioned above. In our application, we use the current location of the user to suggest the available services in that particular area, which are segregated based on the particular category selected.

\section{WORKING:}

The user login activity includes entering the basic credentials like email and password for login purposes. If he is a new user he has to go to the user signup page, and if he is a service provider he should go to service provider sign up page. The users' and the service providers' data will be stored, validated and authenticated using firebase.

Proceedings of the $3^{\text {rd }}$ National Conference on Image Processing, Computing, Communication, Networking and Data Analytics (NCICCNDA 2018) 
Quick Dr: A Smart Mobile Application for Health Care

Then the user can select the particular main category, sub-category and the specialization of his choice and then from that activity he will be directed to the map, and then based on his current location, the chosen category will be displayed, where he can get all the information (address, phone number, ratings, reviews, timings) from selecting a particular marker, which includes Doctors, Hospitals, Pharmacy, Pathology, Blood-Bank, and Ambulance, there-by helping people to get to know, all the details about all the nearby healthcare utilities.

The Special features of our application is,

- It is very user-friendly application where one can use the application without any hindrance.

- It fetches the current location of the user and lists the near-by services that he has requested.

- This application provides wide verity of options in doctor category which allows the user to have wide options.

- Specialization is added in the Allopathy category so that the user will have the option to choose the required one.

- It also provides detailed information about each category, where it provides detailed information like address, phone number, ratings, reviews, working hours etc.

- User can also fix up the appointment with the doctor.

- It also gives the information about the pharmacies which are providing the rarest medicines, discounts.

- It gives information regarding the packages available in pathology laboratories.

- In this application user can add the rating and reviews of the services that he has chosen.

\section{User Login}

This activity mainly focuses on the flow of the application. Once the user gives valid user ID and password, it will be verified and if it is correct he can login to the application. Also if he is a new user he will be directed to the user sign up page, if he is a service provider he will be directed to service provider sign up page on click of respective buttons.
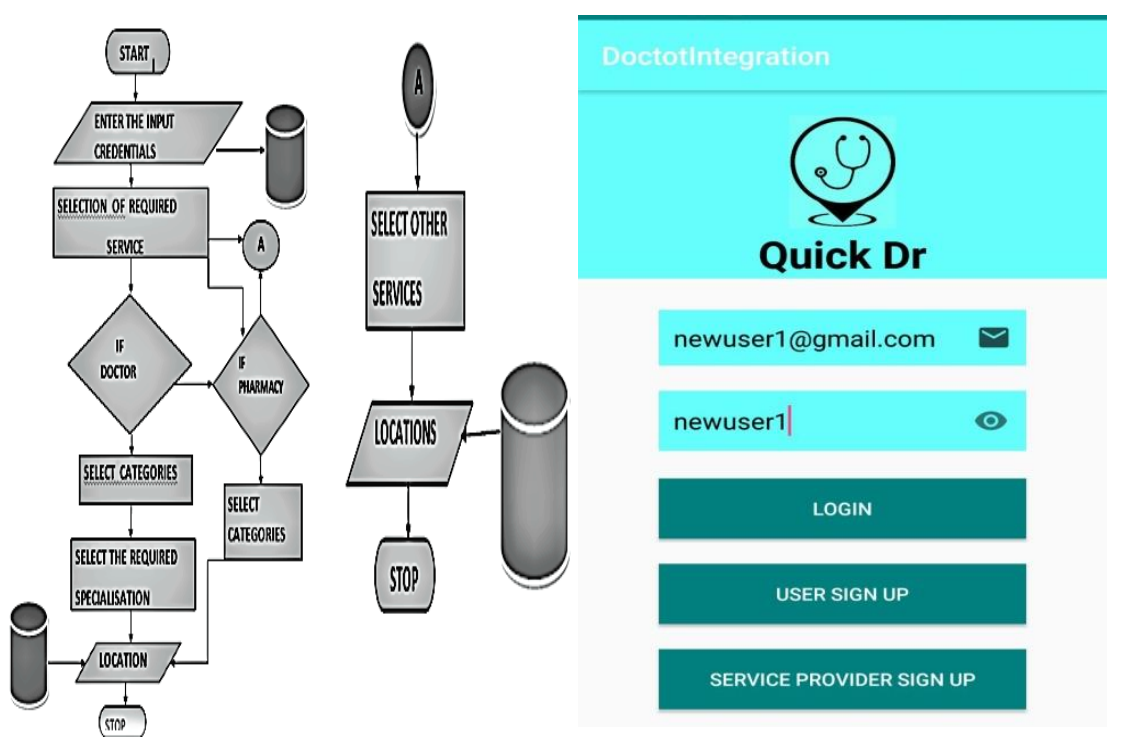

FIG.1 Dataflow diagram for Login. Snapshot.1a User login page

ISBN: 978-81-936820-0-5 34 Series: AIJR Proceedings Proceedings DOI: 10.21467/proceedings. 1 


\section{NEW USER SIGN-UP:}

If a new user wants to use the application, he can do by clicking on the sign-up button. Then he has to fill the necessary details and his details will be stored in the database and his email will be authenticated by firebase. And can use the same user ID and password for next use.

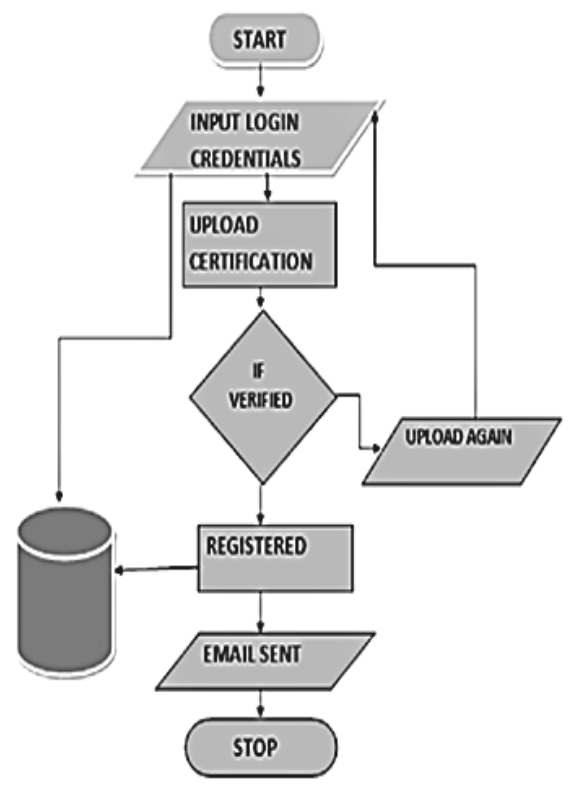

FIG.2. Dataflow diagram for User Sign up
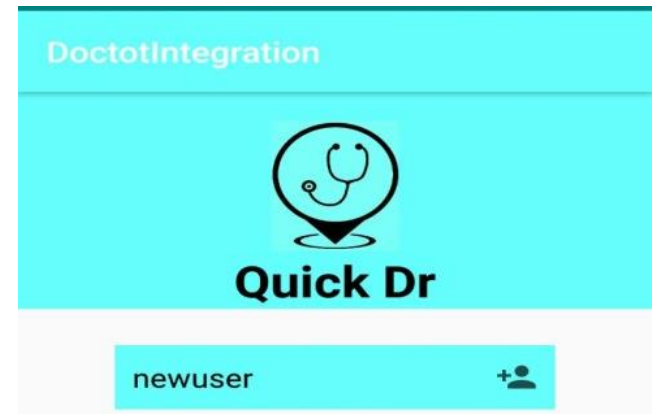

newuser1@gmail.com

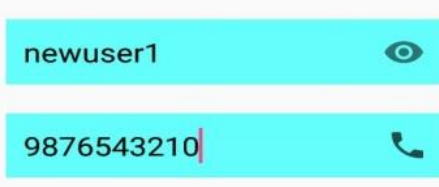

SIGN UP

Snapshot 2b User Sig-UP

Proceedings of the $3^{\text {rd }}$ National Conference on Image Processing, Computing, Communication, Networking and Data Analytics (NCICCNDA 2018) 
Quick Dr: A Smart Mobile Application for Health Care

Service providers are those who provide services to the users which include Hospitals, Doctors, Pharmacy, Pathology, Ambulance, Blood bank. In the sign-up activity he has to select the specific category that he belongs to, from the spinner. And his information will be stored in the database where it will be verified and authenticated.

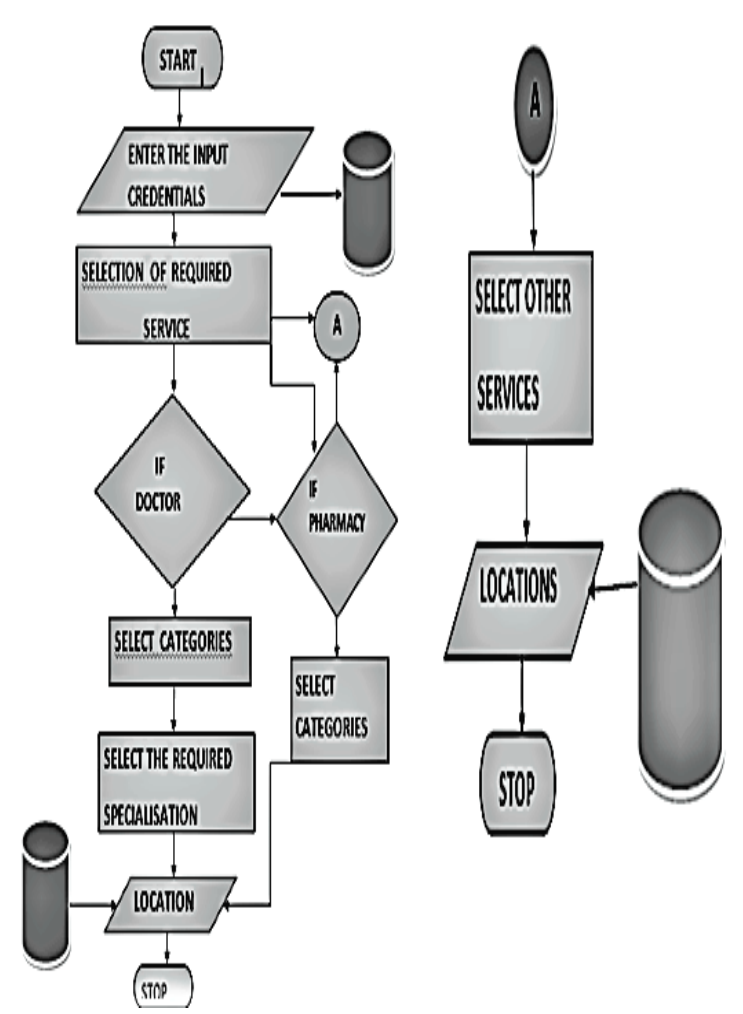

FIG.3. Dataflow diagram for Service Provider Sign Up

\subsection{NAVIGATION PANE}

This activity allows a user to select one of the particular categories provided by the menu and directs the user to the respected screen of his choice.
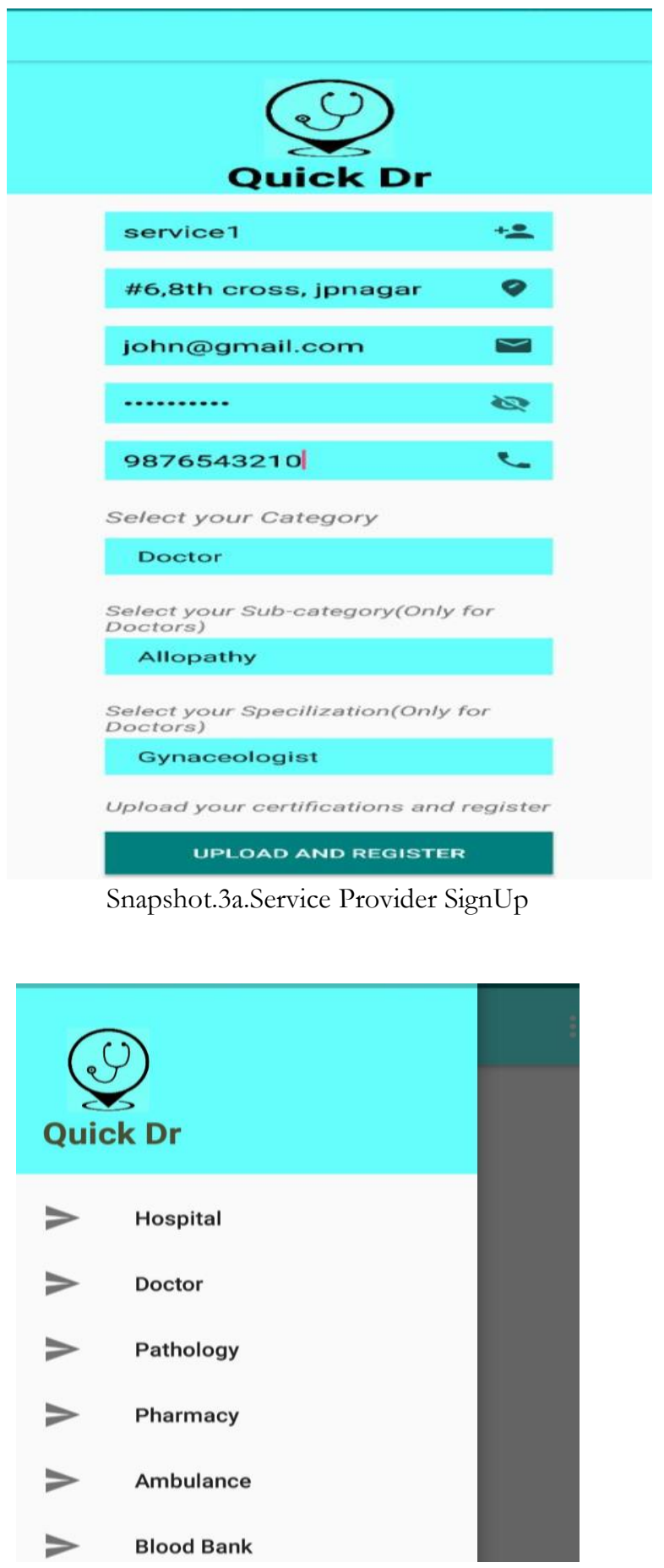

Snapshot.3b.Category Selection Page 


\subsection{GRID LAYOUT}

This activity allows the user to select the specialization of his choice from the allopath category. In this we have included 10 major specializations by choosing the specialization of user choice the activity will be directed to map activity. This activity will be directed to the user after choosing the category of his choice in the navigation pane, and then user will be directed to this activity where his current location will be popped-up along with the near-by category of his choice.

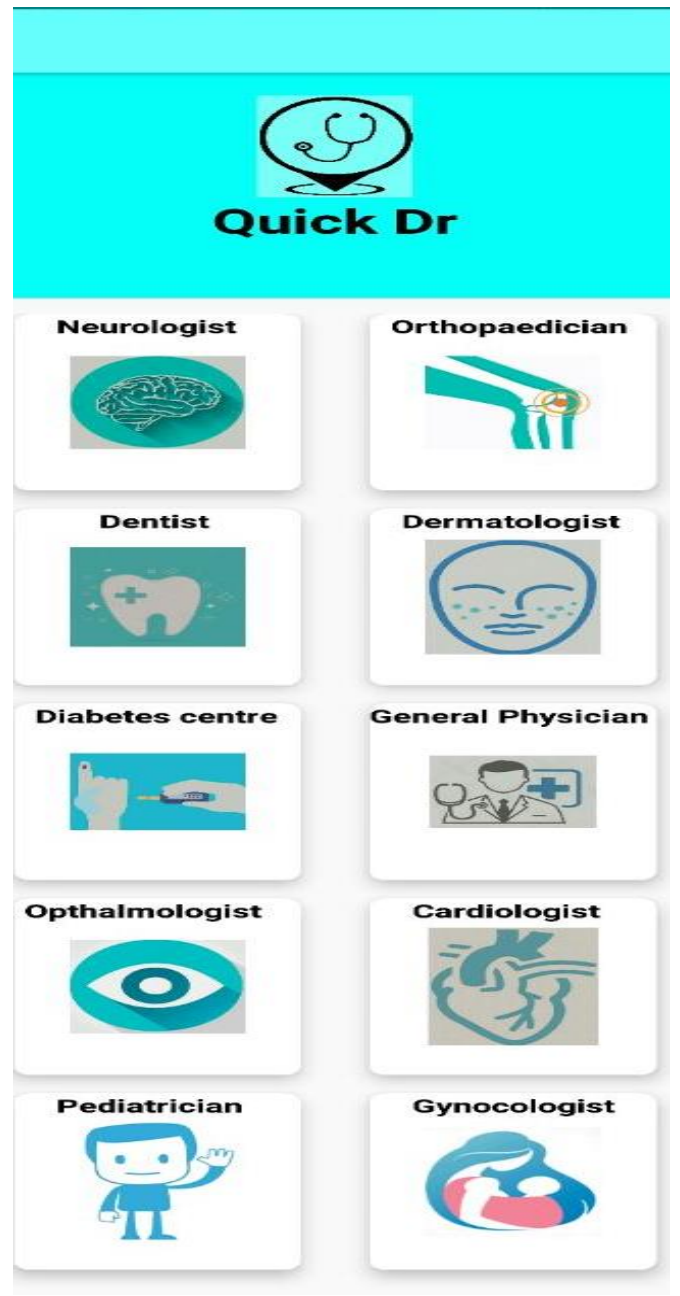

Snapshot.3c.Specialisation Activity

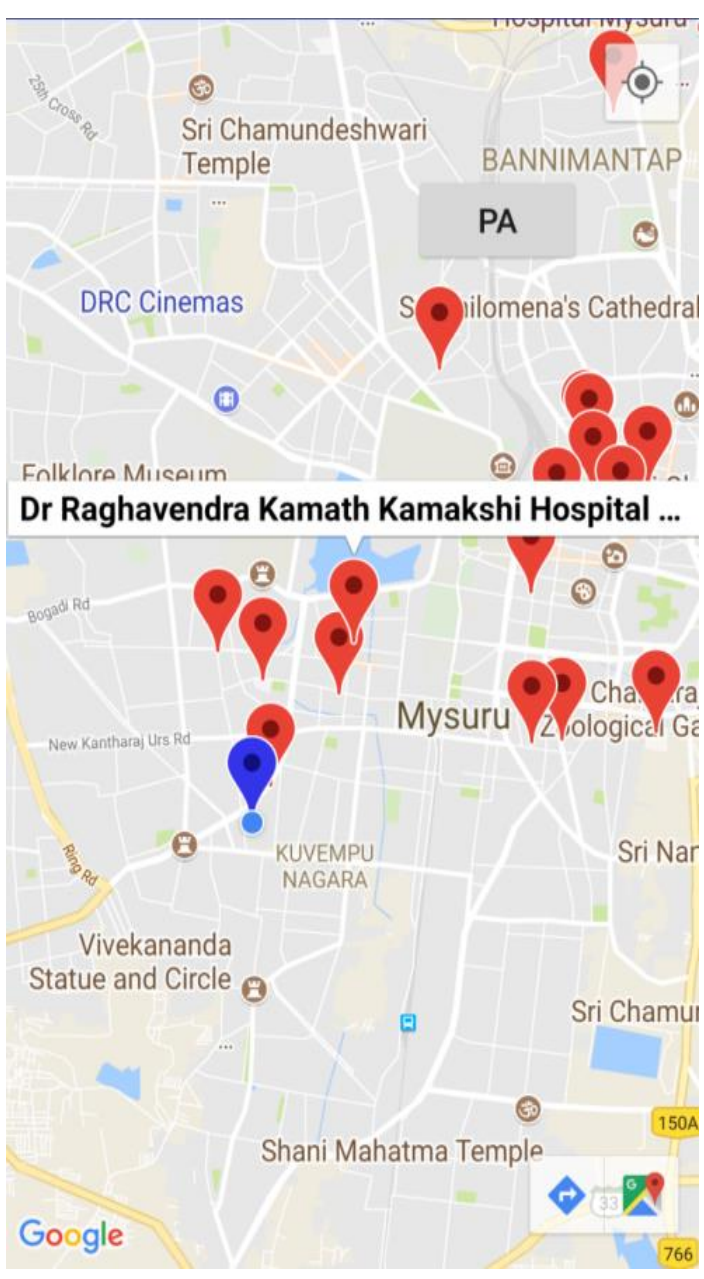

Snapshot.3d.Location of Hospitals

\section{CONCLUSIONS}

By using this application, user can acquire detailed information regarding health care. It provides total assistance for the user throughout the process of getting the medical attention. This mobile application can be modified with a web interface that helps to create a well-built web application. A statistical data can be recorded as and when a user interacts with the

Proceedings of the $3^{\text {rd }}$ National Conference on Image Processing, Computing, Communication, Networking and Data Analytics (NCICCNDA 2018) 
Quick Dr: A Smart Mobile Application for Health Care

application since it uses the GPS to track user's current location, suggested services will be very near and easily accessible to the user.

\section{References}

[1] About an Android Application [online], Available: https://play.google.com/store/apps/details?id=com.practo.fabric [Accessed: 18-February-2018].

[2] About an Android Application related to Online Doctor Consultation [Online], Available: https://play.google.com/store/apps/details?id=com.mfine [Accessed: 19-Febraury-2018].

[3] About an Android Mobile Application to search near-by Hospitals, Doctors [Online], Available: https://play.google.com/store/apps/details?id=com.practo.fabric [Accessed: 20 -February- 2018].

[4] About an Android Application to search pharmacy in the current Location [Online], Available: https:// https://play.google.com/store [Accessed: 21-February- 2018]

[5] About an Android Application to find HealthCare centers near-by the user [Online], Available: https://play.google.com/store/apps/details?id=com.aranoah.healthkart.plus [Accessed: 21 - February- 2018].

[6] About an Android Application to find pathology and hospitals near-by user [Online], Available: https://play.google.com/store/apps/details?id=com.zoylo.com [Accessed: 22-February-2018].

[7] Information about Android Studio [Online], Available: https://developer.android.com/studio/index.html [Accessed: 5March-2018].

[8] Information Firebase Database [Online], Available: https://firebase.google.com/docs/android/setup [Accessed: 5March-2018]. 